\title{
Descrição da atuação do farmacêutico em equipe multiprofissional com ênfase no cuidado ao idoso hospitalizado
}

\author{
Description of the role of the pharmacist in a multiprofessional team focused on the care of \\ hospitalized elderly
}

Isabela Vaz Leite Pinto'

Mariza dos Santos Castro² Adriano Max Moreira Reis ${ }^{2}$

\section{Resumo}

Objetivos: Descrever a atuação do farmacêutico numa equipe multiprofissional, com ênfase no cuidado ao idoso hospitalizado, e intervenções farmacêuticas realizadas durante o seguimento farmacoterapêutico dos indivíduos atendidos. Métodos: Estudo descritivo, exploratório, retrospectivo. A amostra englobou 24 pacientes com idade $\geq 60$ anos, atendidos pelo farmacêutico da equipe multiprofissional de cuidado ao idoso do Hospital Risoleta Tolentino Neves, Belo Horizonte, MG. A farmacoterapia foi analisada em relação ao número de medicamentos; indicação; dose; posologia; aspectos biofarmacêuticos relacionados à via de administração oral ou enteral; estabilidade; incompatibilidade e via de medicamentos parenterais; interações medicamentosas; efetividade e segurança. Verificou-se também a adequação dos medicamentos para idosos, segundo os critérios de Beers. Os medicamentos com atividade anticolinérgica foram identificados segundo Rudolph et al. e Chew et al. As informações foram registradas em instrumento de coleta de dados e a análise estatística descritiva foi realizada em SPSS 10.0. Resultados: Houve diminuição na utilização de medicamentos potencialmente inapropriados para idosos, sendo que na prescrição de alta nenhum idoso estava em uso destes; oito (30\%) utilizaram esses medicamentos na admissão; e cinco $(20,8 \%)$, durante a internação hospitalar. O número de pacientes com prescrição de medicamentos anticolinérgicos reduziu-se de 19 (79,2\%) durante a internação para quatro $(16,6 \%)$ no momento da alta. Vinte e um $(87,5 \%)$ pacientes demandaram intervenções farmacêuticas, sendo que 20 (83,3\%) demandaram intervenções farmacêuticas junto ao médico. Realizou-se um total de 163 intervenções farmacêuticas, com uma média de 6,79 intervenções/paciente e aceitação de 82,2\%. Conclusões: A atuação farmacêutica no cuidado ao idoso, integrada com ações multidisciplinares, otimiza a farmacoterapia, refletindo na segurança e efetividade da assistência prestada ao idoso.

\footnotetext{
Residência Multiprofissional em Saúde do Idoso, Hospital das Clínicas. Universidade Federal de Minas Gerais. Belo Horizonte, MG, Brasil.

2 Departamento de Produtos Farmacêuticos, Faculdade de Farmácia. Universidade Federal de Minas Gerais. Belo Horizonte, MG, Brasil.

Correspondência / Correspondence

Isabela Vaz Leite Pinto

E-mail: isabelaleitepinto@yahoo.com.br
}

Palavras-chave:

Farmacoterapia. Equipe de Assistência ao Paciente. Idoso. Saúde do Idoso. Assistência Hospitalar. 
Abstract

Objective: To describe the role of pharmacists within a multiprofessional team, focusing on care of hospitalized elderly, and pharmacist interventions performed during pharmacotherapy follow up of individuals assisted. Method: Descriptive, exploratory and retrospective study. The sample involved 24 patients aged $\geq 60$ years under the care of the pharmacist in the multiprofessional team for elderly care in the investigated hospital. Pharmacotherapy was analyzed in relation to the number of drugs, their indication, dosage, biopharmaceutical aspects related to the oral or enteral route of administration, stability, incompatibility and parenteral drug routes, drug interactions, effectiveness and safety. The adequacy to the elderly was analyzed according to Beers criteria. Drugs with anticholinergic activity were identified according to Rudolph et al. and Chew et al. Information was recorded on a data collection tool and the descriptive statistic analysis was carried out in SPSS 10.0. Results: At the moment of hospital discharge, none of the subjects were making use of drugs potentially inappropriate for the elderly; eight $(30.0 \%)$ patients were using such drugs when admitted to hospital; and five $(20.8 \%)$ used such drugs during their hospitalization. The number of patients in use of anticholinergic drugs decreased from $19(79.2 \%)$ during hospitalization to four $(16.6 \%)$ at hospital discharge. Twenty one (87.5\%) patients required pharmaceutical interventions and $20(83.3 \%)$ required pharmaceutical interventions by the physician. A total of 163 pharmaceutical interventions were carried out, representing a mean of 6.79 interventions/patient and $82.2 \%$ acceptance. Conclusion: The role of pharmacists in caring for elderly optimizes pharmacotherapy and affects the safety and effectiveness of the aged care.
Key words: Drug Therapy. Patient Care Team. Aged. Health of the Elderly. Hospital Care.

\section{INTRODUÇÃO}

$\mathrm{Na}$ população idosa, são frequentes as múltiplas condições crônicas de saúde e o uso elevado de medicamentos que levam à polifarmácia, definida como o uso concomitante de cinco ou mais medicamentos. ${ }^{1-3} \mathrm{~A}$ polifarmácia pode levar a não adesão ao tratamento, reações adversas a medicamentos, interações medicamento-medicamento, maior risco de erros de medicação, risco de quedas, aumento da taxa de hospitalização, entre outros resultados terapêuticos negativos. ${ }^{2,3}$ Aliado a esse fenômeno, observa-se que o idoso possui necessidades físicas e sociais complexas, necessitando de cuidado com foco na colaboração multiprofissional. ${ }^{4}$

A colaboração profissional requer ou promove relações e interações nas quais os profissionais poderão partilhar conhecimentos, especialização e habilidades entre si, com o objetivo de proporcionar melhor atenção ao paciente..$^{5} \mathrm{O}$ cuidado multidisciplinar ao idoso responde às necessidades complexas desta população, ao lidar com as comorbidades, melhorar os processos de saúde e resultados relacionados às síndromes geriátricas. ${ }^{4}$

À luz da complexidade do manejo da farmacoterapia em pacientes idosos, o farmacêutico tem sido incluído em equipes multiprofissionais de cuidado ao idoso, devido a seu conhecimento acerca dos medicamentos. ${ }^{6}$ O cuidado farmacêutico no contexto de uma equipe multiprofissional geriátrica melhorou a qualidade do uso de medicamentos durante a internação e após a alta hospitalar, diminuindo riscos e melhorando os resultados terapêuticos. ${ }^{7}$

Revisão sistemática sobre a atuação do farmacêutico em equipes multiprofissionais nos EUA evidenciou efeitos favoráveis da atuação desse profissional no cuidado direto ao paciente em termos terapêuticos, humanísticos e relativos à segurança. ${ }^{8}$ No Brasil, estudos abordando a atuação do farmacêutico na farmacoterapia de idosos são escassos, especialmente no âmbito hospitalar. $^{9-11}$ 
A atuação do farmacêutico na promoção do uso racional de medicamentos por idosos e sua inserção em equipes multiprofissionais otimizam a farmacoterapia dos pacientes geriátricos e ampliam a qualidade e segurança do cuidado. ${ }^{67,11}$ Nesse contexto, o artigo visou descrever as atividades desenvolvidas pelo farmacêutico numa equipe multiprofissional, com ênfase no cuidado ao idoso hospitalizado e as intervenções farmacêuticas realizadas durante o seguimento farmacoterapêutico dos indivíduos atendidos.

\section{METODOLOGIA}

\section{Delineamento do estudo}

Estudo descritivo, exploratório e retrospectivo da atuação do farmacêutico em uma equipe multidisciplinar de atenção ao idoso hospitalizado.

\section{Caracterização do local do estudo}

O estudo foi desenvolvido no Hospital Risoleta Tolentino Neves (HRTN), um hospital geral, público, de ensino, com cerca de 310 leitos, integrado ao sistema de atenção a urgência e emergência da região metropolitana de Belo Horizonte, MG.

\section{População estudada}

A população do estudo foi composta por indivíduos com idade igual ou maior de 60 anos, sem distinção de gênero, atendidos pelo farmacêutico da equipe multiprofissional, com ênfase no cuidado ao idoso do HRTN, no período de setembro de 2010 a janeiro de 2011. O trabalho da equipe multiprofissional de atenção ao idoso era prestado aos pacientes internados que preenchiam os perfis de risco - baseados nas comorbidades: acidente vascular encefálico (AVE), insuficiência cardíaca (IC), demência, fratura e insuficiência vascular. A amostra não probabilística englobou 24 pacientes que preencheram os critérios de inclusão.
Equipe multiprofissional de atenção ao idoso

Considerando-se a necessidade de aprimorar o cuidado ao idoso com foco na colaboração multiprofissional, em setembro de 2010 foi estruturado um trabalho interativo entre os residentes multiprofissionais em saúde do idoso (enfermeiro, farmacêutico, fisioterapeuta, fonoaudiólogo, dentista e terapeuta ocupacional) e profissionais do hospital (geriatra, nutricionista, assistente social e psicólogo).

O paciente era atendido por cada um dos profissionais da equipe, conforme a demanda observada pelos profissionais. A equipe multiprofissional se reunia duas vezes na semana para discutir o caso dos pacientes e planejar o cuidado. O plano de cuidados de cada paciente englobava os aspectos clínicos e sociais e era discutido pela equipe multiprofissional. Os aspectos farmacoterápicos eram analisados pelo farmacêutico na perspectiva da necessidade, segurança e efetividade, de forma integrada com a equipe multiprofissional. Além disso, a equipe preocupava-se com o referenciamento do paciente na rede do Sistema Único de Saúde (SUS). Foi um trabalho pioneiro no hospital e de extrema importância para o cuidado ao idoso, pois este era visto a partir de diversos olhares, de forma global, o que contribuiu para a integralidade do cuidado.

Atividades do farmacêutico na equipe multiprofissional de cuidado ao idoso

O seguimento farmacoterápico dos pacientes realizado pelo farmacêutico apresentava as seguintes etapas: revisão da prescrição médica, exames laboratoriais e evolução clínica registrados no prontuário; entrevista ao paciente e/ou cuidador; elaboração da anamnese farmacológica; análise da farmacoterapia e elaboração do plano de cuidado e intervenções farmacêuticas. Após a coleta de dados no prontuário e entrevista ao paciente, o farmacêutico realizava a análise da farmacoterapia, considerando os seguintes aspectos: indicação; efetividade; segurança; dose; posologia; aspectos biofarmacêuticos 
relacionados à via de administração oral ou enteral; estabilidade da formulação; incompatibilidade físico-química entre medicamentos parenterais e interações medicamentosas.

Feita a análise dos aspectos farmacoterápicos, em caso de necessidade, eram sugeridas intervenções pelo farmacêutico, na perspectiva de contribuir para um tratamento medicamentoso seguro e efetivo. As intervenções eram comunicadas durante as reuniões da equipe ou em visitas clínicas de avaliação e eram registradas no prontuário. As informações coletadas diretamente do paciente/cuidador, e as intervenções farmacêuticas realizadas também eram documentadas no prontuário.

Antes da alta hospitalar, o paciente era orientado pelo farmacêutico sobre a terapia medicamentosa a ser realizada em domicílio, enfatizando orientações sobre o acesso ao medicamento na rede de saúde e os esquemas posológicos a serem seguidos. Nessa atividade, o farmacêutico usava estratégias de comunicação oral e escrita, adequadas ao nível de compreensão de cada indivíduo. Uma semana após a alta, o paciente era contatado pelo farmacêutico via telefone, para obter informações sobre acesso, adesão, segurança (ocorrência de reações adversas) e efetividade dos medicamentos. Mediante identificação da existência ou probabilidade da existência de problemas relacionados ao uso de medicamentos, eram realizadas intervenções farmacêuticas.

\section{Coleta de dados}

As informações relativas às características demográficas e clínicas dos pacientes, à farmacoterapia e às intervenções realizadas pelo farmacêutico, foram coletadas nos prontuários e registradas em um instrumento estruturado de coleta de dados. Na operacionalização da coleta de dados, utilizaram-se as seguintes definições:

Indice de comorbidade de Charlson - método de classificação de gravidade que utiliza dados dos diagnósticos secundários e da idade. Foi calculado empregando as informações clínicas disponíveis no prontuário e preconizadas por Charlson et al. ${ }^{12}$

Medicamento potencialmente inapropriado para idoso (MIP) - definido como qualquer medicamento cujos riscos potenciais superam os benefícios esperados. Assim, tais medicamentos devem ser evitados na farmacoterapia dos idosos..$^{13}$ Os medicamentos utilizados pelo paciente nos períodos pré-internação (uso domiciliar), durante a internação e prescritos para alta hospitalar foram identificados e classificados como MIP, de acordo com os critérios definidos por Fick et al. ${ }^{14}$

Medicamentos com atividade anticolinérgica medicamento cuja atividade se opõe ou bloqueia a ação da acetilcolina. $\mathrm{O}$ uso de medicamentos com atividade anticolinérgica em idosos tem sido frequentemente citado na literatura como causa de eventos adversos como quedas, comportamento impulsivo, perda da independência, disfunção cognitiva, etc. ${ }^{15}$ Desta maneira, os medicamentos utilizados em domicílio, os prescritos na internação e na alta foram classificados em relação à atividade anticolinérgica, de acordo com Rudolph et al..$^{15}$ e Chew et al. ${ }^{16}$

O número de medicamentos prescritos pelos idosos foi estratificado nas seguintes categorias: oligofarmácia - menor ou igual a quatro medicamentos $;{ }^{17}$ polifarmácia - maior ou igual a cinco medicamentos; ${ }^{2,17}$ polifarmácia em idosos - seis a nove medicamentos ${ }^{2}$ e polifarmácia excessiva - maior ou igual a 10 medicamentos. ${ }^{2}$

Análise estatística

Desenvolveu-se análise estatística descritiva empregando o software Statistical Package for Social Sciences, SPSS 10.0, compreendendo determinação de frequência absoluta e relativa, média e desviopadrão.

\section{Procedimentos éticos}

A pesquisa foi conduzida de acordo com a Resolução no 196/96 do Conselho Nacional de Saúde e aprovada pelo Comitê de Ética em 
Pesquisa da Universidade Federal de Minas Gerais, sob n ETIC 0237.0.203.000-11.

\section{RESULTADOS}

No período da investigação, 24 pacientes foram acompanhados pelo farmacêutico da equipe multiprofissional de cuidado ao idoso. Os pacientes atendidos pelo farmacêutico apresentaram média de idade de 74,6 anos $(\mathrm{dp}=10,0)$, sendo que $66,6 \%$ dos pacientes tinham idade maior que 70 anos; 19 $(79,2 \%)$ pacientes eram do sexo feminino. A média do tempo de internação foi de $23,2(\mathrm{dp}=19,2)$ dias, e do índice de comorbidade de Charlson, 5,3 $(\mathrm{dp}=1,6)($ tabela 1$)$.

Os perfis de risco que determinaram o maior número de pacientes encaminhados para monitorização foram fratura e insuficiência cardíaca, e em menor proporção, demência (tabela 1). Cinco pacientes atendidos apresentavam várias patologias e, por isso, podiam ser incluídos em mais de um perfil de risco. Neste caso, o paciente foi incluído no perfil de risco da condição clínica que determinou a internação hospitalar.

Tabela 1 - Caracterização sociodemográfica e clínica dos pacientes $(n=24)$ atendidos pelo farmacêutico da equipe multiprofissional. Belo Horizonte, MG, 2010-2011.

\begin{tabular}{lc}
\hline \multicolumn{1}{c}{ Característica } & Valor \\
\hline Gênero [n (\%)] & \\
$\quad$ Masculino & {$[5(20,8 \%)]$} \\
$\quad$ Feminino & {$[19(79,2 \%)]$} \\
Idade [média (desvio-padrão)] & {$[74,6(10,0)]$} \\
Tempo de internação em dias [média (desvio-padrão)] & {$[23,2(19,2)]$} \\
Índice de comorbidade de Charlson [média (desvio-padrão)] & {$[5,3(1,6)]$} \\
Perfil de risco principal [n (\%)] & \\
$\quad$ Fratura & {$[8(33,3 \%)]$} \\
Insuficiência cardíaca & {$[8(33,3 \%)]$} \\
Cirurgia vascular & {$[4(16,7 \%)]$} \\
Acidente vascular encefálico & {$[3(12,5 \%)]$} \\
Demência & {$[1(4,2 \%)]$} \\
Número de perfis de risco/paciente [n (\%)] & \\
1 perfil de risco & {$[19(79,2 \%)]$} \\
2 perfis de risco & {$[5(20,8 \%)]$} \\
Desfecho [n (\%)] & \\
Alta hospitalar & {$[19(79,2 \%)]$} \\
Óbito & {$[4(16,7 \%)]$} \\
Transferência para outro hospital & {$[1(4,2 \%)]$} \\
\hline
\end{tabular}

A média do número de medicamentos utilizados antes da internação foi de 4,48 $(\mathrm{dp}=3,20)$. Já após a internação hospitalar, a média do número de medicamentos usados foi de 6,32 (dp=2,43). Os números de medicamentos utilizados pelos idosos, estratificados segundo os valores que definem as categorias de polifarmácia, são apresentados na tabela 2 . 
Tabela 2 - Número de medicamentos utilizados pelos pacientes atendidos pelo farmacêutico da equipe multidisciplinar antes e após a internação hospitalar. Belo Horizonte, MG, 2010-2011.

\begin{tabular}{lcc}
\hline \multicolumn{1}{c}{ Número de medicamentos utilizados } & Frequência absoluta & Porcentagem (\%) \\
\hline \multicolumn{1}{c}{ Antes da internação hospitalar } & 12 & 50 \\
Oligofarmácia (0 a 4 medicamentos) & 3 & 12,5 \\
Polifarmácia (5 a 6 medicamentos) & 3 & 12,5 \\
Polifarmácia em idosos (6 a 9 medicamentos) & 3 & 12,5 \\
Polifarmácia excessiva ( $\geq 10$ medicamentos) & 3 & 12,5 \\
$\quad$ Informação não disponível & & \\
Após a internação hospitalar & 4 & 16,7 \\
Oligofarmácia (0 a 4 medicamentos) & 7 & 29,2 \\
Polifarmácia (5 a 6 medicamentos) & 4 & 16,7 \\
Polifarmácia em idosos (6 a 9 medicamentos) & 2 & 8,3 \\
Polifarmácia excessiva ( $\geq 10$ medicamentos) & 7 & 29,2 \\
$\quad$ Informação não disponível & & \\
\hline
\end{tabular}

Com relação ao uso de medicamentos internação hospitalar, quando comparada ao potencialmente inapropriados para idosos, período antes da internação (tabela 3). observou-se menor frequência durante a

Tabela 3 - Idosos em uso de medicamentos classificados como inapropriados para idosos, segundo Fick et al., ${ }^{14}$ atendidos pelo farmacêutico da equipe multiprofissional antes, durante e após a internação hospitalar. Belo Horizonte, MG, 2010-2011.

\begin{tabular}{llcc}
\hline \multicolumn{1}{c}{$\begin{array}{c}\text { Momento da } \\
\text { utilização }\end{array}$} & \multicolumn{1}{c}{ Medicamentos } & $\begin{array}{c}\text { Frequência } \\
\text { absoluta }\end{array}$ & $\begin{array}{c}\text { Porcentagem } \\
(\%)\end{array}$ \\
\hline $\begin{array}{l}\text { Antes da internação } \\
\text { hospitalar }\end{array}$ & $\begin{array}{l}\text { Digoxina }(>0,125 \mathrm{mg} / \text { dia), Prometazina, } \\
\text { Clonidina, Hidroxizina, Diazepam, } \\
\text { Fluoxetina, Metildopa } \\
\text { Óleo mineral, Amiodarona, } \\
\text { Nurante a internação } \\
\text { hospitalar }\end{array}$ & 8 & 30,0 \\
$\begin{array}{l}\text { Após a internação } \\
\text { hospitalar }\end{array}$ & - & 5 & 20,8 \\
\hline
\end{tabular}

Nas prescrições de pacientes em alta hospitalar, não foi identificado nenhum paciente em uso de medicamentos potencialmente inapropriados para idosos. A proporção de pacientes em uso de medicamentos anticolinérgicos foi consideravelmente maior durante a internação hospitalar do que nos períodos antes e após a internação (tabela 4). 
Tabela 4 - Idosos em uso de medicamentos com atividade anticolinérgica antes, durante e após a internação hospitalar, atendidos pelo farmacêutico da equipe multiprofissional. Belo Horizonte, MG, 2010-2011.

\begin{tabular}{llcc}
\hline \multicolumn{1}{c}{$\begin{array}{c}\text { Momento da } \\
\text { utilização }\end{array}$} & \multicolumn{1}{c}{ Medicamento } & $\begin{array}{c}\text { Frequência } \\
\text { absoluta }\end{array}$ & $\begin{array}{c}\text { Porcentagem } \\
(\%)\end{array}$ \\
\hline $\begin{array}{l}\text { Antes da internação } \\
\text { hospitalar }\end{array}$ & Prometazina, Hidroxizina, Flunarizina & 2 & 8,3 \\
$\begin{array}{l}\text { Durante a internação } \\
\text { hospitalar }\end{array}$ & $\begin{array}{l}\text { Metoclopramida, Haloperidol, } \\
\text { Mirtazapina, N-butilbrometo de } \\
\text { escopolamina, Ipratrópio }\end{array}$ & 19 & 79,2 \\
$\begin{array}{l}\text { Após a internação } \\
\text { hospitalar }\end{array}$ & Haloperidol, Trazodona, Ipratrópio & 4 & 16,6 \\
\hline
\end{tabular}

Dos 24 pacientes submetidos ao seguimento farmacoterápico, $21 \quad(87,5 \%)$ demandaram intervenções farmacêuticas. As intervenções farmacêuticas realizadas em integração com a equipe multiprofissional são apresentadas na tabela 5 .

Tabela 5 - Intervenções farmacêuticas realizadas junto aos pacientes atendidos pelo farmacêutico da equipe multiprofissional. Belo Horizonte, MG, 2010-2011.

\begin{tabular}{lcc}
\hline \multicolumn{1}{c}{ Intervenções } & $\begin{array}{c}\text { Frequência } \\
\text { absoluta }\end{array}$ & $\begin{array}{c}\text { Porcentagem } \\
(\%)\end{array}$ \\
\hline $\begin{array}{l}\text { Intervenções realizadas pelo farmacêutico } \\
\text { Intervenções farmacêuticas realizadas em integração com a equipe } \\
\text { multiprofissional }\end{array}$ & 21 & 87,5 \\
$\quad$ Médico & 20 & 83,3 \\
$\quad$ Enfermeiro & 10 & 41,7 \\
$\quad \begin{array}{l}\text { Demais profissionais da equipe (assistente social, dentista, } \\
\text { fisioterapeuta, fonoaudiólogo, nutricionista, psicólogo e }\end{array}$ & 4 & 16,7 \\
terapeuta ocupacional) & & \\
Intervenções farmacêuticas realizadas com paciente e/ou familiares & 11 & 45,8 \\
\hline
\end{tabular}

Realizou-se um total de 163 intervenções farmacêuticas, com média de 6,79 (dp=7,6) intervenções por paciente e aceitação de $82,2 \%$
( $\mathrm{n}=134)$. Exemplos de intervenções realizadas pelo farmacêutico são apresentadas no quadro 1. 
Quadro 1 - Exemplos de intervenções farmacêuticas no seguimento farmacoterapêutico de idosos em equipe multiprofissional. Belo Horizonte, MG, 2010-2011.

Sugestão de suspensão do uso de medicamentos inapropriados para idoso; por exemplo, óleo mineral por via oral.

Sugestão de inclusão de algum medicamento necessário à farmacoterapia.

Sugestão de troca de algum medicamento por outro mais adequado; por exemplo, sugestão de troca de clonazepam por lorazepam devido ao risco de queda.

Sugestão de adequação da farmacoterapia de acordo com a disponibilidade na atenção básica.

Ajuste posológico de medicamentos para pacientes com injúria renal aguda ou doença renal crônica.

Monitorização terapêutica de digoxina.

Controle da razão normalizada internacional - RNI e análise de interações medicamentosas em pacientes em uso de varfarina.

Orientação da equipe quanto ao risco e à ocorrência de interações medicamento x medicamento;

por exemplo, interação medicamentosa entre cloreto de potássio e inibidor da enzima conversora da angiotensina.

Orientação da equipe quanto à administração de medicamentos via sonda nasoentérica (SNE) e risco de interações com nutrição enteral; por exemplo, uso de omeprazol por SNE.

Orientação da equipe quanto ao risco e à ocorrência de reações adversas a medicamento.

Orientação dos profissionais de enfermagem sobre recomendações para administração de medicamentos parenterais.

Educação em saúde a pacientes e familiares a respeito do uso de medicamentos e necessidade de adesão à terapia medicamentosa.

Encaminhamento dos pacientes a outros profissionais da equipe multidisciplinar e serviços de saúde ambulatoriais.

Orientações farmacêuticas na alta hospitalar foram realizadas para 11 pacientes, que representam 57,9\% dos 19 pacientes cujo desfecho foi a alta hospitalar. As orientações eram relacionadas a acesso, adesão, compreensão do tratamento e técnicas de utilização de medicamentos.

\section{DISCUSSÃO}

A elevada frequência de intervenções desenvolvidas diretamente pelo farmacêutico junto ao paciente e de forma integrada com a equipe multiprofissional, identificada no presente estudo, demonstra a relevância da atuação do profissional farmacêutico em uma equipe multiprofissional com ênfase ao cuidado ao idoso hospitalizado. Em muitos estudos presentes na literatura, há abordagem sobre a atuação do farmacêutico em idosos que vivem em instituições de longa permanência ${ }^{18}$ e em idosos da comunidade. 11,19,20 Entretanto, a produção científica sobre a atuação do farmacêutico em equipe multiprofissional ao idoso hospitalizado, até o presente momento, é incipiente. ${ }^{6,21-24}$

Os estudos nacionais relacionados ao seguimento farmacoterápico e à intervenção do farmacêutico para promoção do uso racional de medicamentos em idosos são escassos, desenvolvidos na comunidade e direcionados a portadores de doenças específicas, ${ }^{10,11}$ não havendo estudos relativos à atuação do farmacêutico ao idoso no ambiente hospitalar. 
A equipe multiprofissional envolvida nesta investigação tem composição ampliada, o que permite uma avaliação multidimensional do idoso e um planejamento integrado do plano de cuidados. Esse aspecto é essencial no cuidado ao idoso. A presença de um médico na equipe agiliza a discussão e a implementação das intervenções farmacêuticas que, muitas vezes, dependem de modificações na prescrição da farmacoterapia. Este fato é comprovado no presente estudo, em que para 83,3\% ( $\mathrm{n}=20)$ dos pacientes houve intervenções farmacêuticas junto ao médico. Os estudos publicados até o momento abordando intervenções farmacêuticas junto a idosos hospitalizados apresentaram equipes multidisciplinares com diferentes composições, porém a maioria apresenta composição reduzida. Em um estudo, não havia médico na equipe. ${ }^{6}$ Já outros dois estudos, apresentavam a interação do farmacêutico com o médico e o enfermeiro; ${ }^{24}$ em outro, houve apenas a interação do farmacêutico com o médico, ${ }^{23}$ sem indicação da presença de outras profissões como integrantes da equipe multidisciplinar.

A aceitação das intervenções do farmacêutico foi demostrada em estudos anteriores, com valores que variaram de 67 a $92 \% .^{21-26}$ Os estudos de Lam \& Ruby $^{21}$ e Storm ${ }^{24}$ foram os que apresentaram valores de aceitação mais próximos do presente trabalho: $83,9 \%$ e $77 \%$, respectivamente. A aceitação das intervenções do farmacêutico foi $82,2 \%$, inferior ao detectado em outras investigações desenvolvidas no âmbito hospitalar. ${ }^{21,25,26}$ Uma explicação para isso é o fato de o presente estudo referir-se ao período de implantação do serviço de cuidado ao idoso em equipe multiprofissional, quando a inserção do farmacêutico em tal equipe também estava sendo implantada. Devido a isso, pode ter havido resistência inicial por parte dos profissionais da equipe frente às intervenções do farmacêutico, muitas vezes por desconhecerem a atuação desse profissional na área clínica.

No presente estudo, observou-se que a média do número de medicamentos prescritos antes da internação hospitalar foi 4,5 (dp=3,2). O número de medicamentos em uso antes da internação hospitalar na amostra investigada é semelhante ao valor observado em um estudo com idosos da comunidade no Brasil: ${ }^{11}$ 4,4 medicamentos $(\mathrm{dp}=2,9)$. Esse valor, porém, é mais alto do que a utilização de 2,18 medicamentos, identificado em um estudo de base populacional sobre o uso de medicamentos em idosos da região Metropolitana de Belo Horizonte, $\mathrm{MG} .{ }^{27} \mathrm{O}$ valor mais alto encontrado no presente estudo pode ser justificado pelo fato de os idosos incluídos na investigação apresentarem comorbidades, como insuficiência cardíaca, que está associada ao uso de grande número de medicamentos.

A média do número de medicamentos em uso após a internação hospitalar foi de 6,32 (dp=2,43), semelhante ao observado em estudos publicados na literatura, ${ }^{6,21,23}$ demonstrando, assim, que a presença da polifarmácia é evento frequente entre idosos. Observou-se aumento no número de medicamentos em uso e da prevalência de polifarmácia, após a internação hospitalar nos pacientes encaminhados para seguimento pelo farmacêutico. Rollanson \& Vogt, ${ }^{3}$ em revisão sistemática sobre a participação do farmacêutico na redução de polifarmácia em pacientes idosos, encontraram sete $(50 \%)$ estudos que mostraram que a intervenção realizada resultou em redução no número médio de medicamentos em uso, e apenas um $(7,1 \%)$ estudo que mostrou aumento no número médio de medicamentos após a intervenção. Os resultados do presente estudo são divergentes do observado na literatura e uma possível explicação para isso também se baseia nas comorbidades dos pacientes estudados, tendo em vista que o tratamento da insuficiência cardíaca consiste no uso de múltiplos medicamentos. Neste caso, a compensação do paciente é muitas vezes atingida com a prescrição de fármacos de classes terapêuticas diferentes, aumentando o número de medicamentos em uso.

Em relação ao uso de medicamentos inapropriados por idosos (MIP), as intervenções farmacêuticas contribuíram para a redução do seu emprego após a alta hospitalar pelos pacientes incluídos na investigação. Esse 
resultado está em consonância com uma revisão sistemática de estudos de intervenção que tiveram como objetivo reduzir a prescrição de MIP para idosos. Esta revisão demonstrou o impacto das intervenções do farmacêutico e das intervenções multidisciplinares, envolvendo a atuação do farmacêutico na diminuição da prescrição de MIP. ${ }^{28}$

A utilização de MIP leva a um maior risco de desenvolver eventos adversos a medicamentos, e os idosos são mais vulneráveis a desenvolver tais eventos. Assim, a intervenção farmacêutica, ao reduzir a prescrição de MIP, contribui para evitar eventos adversos, resultando em melhores resultados clínicos. Algumas vezes, porém, há a necessidade do uso de MIP por não haver alternativas terapêuticas mais efetivas no mercado e/ou no sistema público de assistência farmacêutica. Como exemplo, há a amiodarona, que em determinadas condições clínicas não apresenta alternativa terapêutica mais segura. Para garantir ao idoso uma farmacoterapia segura, recomendam-se monitorização contínua e uso dos medicamentos adequados em doses adequadas.

O uso de medicamentos com propriedade anticolinérgica está associado a eventos adversos como constipação, boca seca, visão borrada, tontura, retenção urinária, piora da função cognitiva e demência, estando também associado a quedas, delirium e comportamento impulsivo. ${ }^{29}$ Wawruch et al., ${ }^{30}$ em estudo sobre o uso de medicamentos com propriedade anticolinérgica em idosos hospitalizados, demonstraram que a hospitalização leva ao aumento na prevalência do uso de tais medicamentos, comparando o uso dos mesmos na admissão e após a alta hospitalar $(10,5 \%$ e $14,2 \%$, respectivamente; $\mathrm{p}<0,001)$. O mesmo fato foi encontrado no presente estudo, em que o número de pacientes em uso de medicamentos com propriedade anticolinérgica antes da internação era dois $(8,3 \%)$ e, após a alta hospitalar, quatro $(16,6 \%)$.

A prescrição de medicamentos com propriedade anticolinérgica durante a internação hospitalar foi elevada ( $\mathrm{n}=19$ pacientes; 79,2\% dos pacientes) e pode ser explicada pela alta frequência de prescrição de metoclopramida. Todos os pacientes que apresentaram a prescrição de algum medicamento com propriedade anticolinérgica tinham a metoclopramida como um dos medicamentos prescritos. $\mathrm{O}$ fato exposto pode ser explicado pelo padrão de prescrição nos hospitais brasileiros.

Cruciol-Souza et al., ${ }^{31}$ ao avaliarem as prescrições medicamentosas em um hospital universitário brasileiro, detectaram que a metoclopramida estava entre os 10 medicamentos mais prescritos. Braga et al..$^{32}$ também detectaram fato semelhante: a metoclopramida esteve entre um dos cinco medicamentos mais prescritos em um hospital universitário brasileiro. Observa-se, muitas vezes, que a metoclopramida é prescrita como um medicamento "se necessário", ou seja, apenas se o paciente apresentar náuseas ou vômitos esse medicamento será utilizado.

Para otimizar a farmacoterapia do paciente idoso, o farmacêutico, além de monitorar a efetividade da resposta terapêutica, deve identificar eventos adversos a medicamentos e seus determinantes, com ênfase naqueles associados aos fármacos anticolinérgicos e MIP. Entre as intervenções a serem realizadas pelo farmacêutico, consta sugerir ao médico alternativas terapêuticas a esses fármacos, quando disponíveis no mercado e/ou no sistema público de assistência farmacêutica.

O seguimento farmacoterapêutico de idosos, com ações integradas e multidisciplinares, é uma estratégia que aprimora a qualidade da prescrição de medicamentos em Geriatria e evita eventos adversos a medicamentos. Essa nova perspectiva de monitorização da farmacoterapia do paciente idoso é considerada um novo paradigma na atenção a essa faixa etária e uma medida que deve ser incorporada no processo assistencial e no cuidado baseado em evidências. ${ }^{33}$

A presente investigação apresenta as seguintes limitações: o pequeno número de indivíduos acompanhados, a atuação realizada por apenas um farmacêutico e limitada a curto período de tempo. Tais resultados se referem ao período de implantação do serviço de atenção ao idoso em 
equipe multiprofissional no hospital investigado. Desta forma, os resultados não são generalizáveis, mas dados relativos à aceitação das intervenções são semelhantes aos descritos em outros estudos. $^{21,24}$ Sugere-se que estudos controlados sejam realizados posteriormente, para analisar o impacto da atuação do farmacêutico nos resultados clínicos, humanísticos e econômicos relacionados à farmacoterapia. Apesar das limitações, o trabalho é relevante por estudar um tema pouco explorado pela literatura internacional e, até o presente momento, ausente na produção científica brasileira.

\section{CONCLUSÃO}

As atividades clínicas realizadas pelo farmacêutico apresentaram boa aceitação pela equipe multiprofissional e envolveram, principalmente, o profissional médico.

Ao contribuir para a redução do número de medicamentos inapropriados para idosos e sugerir intervenções considerando as particularidades dos pacientes dessa faixa etária, o farmacêutico contribui na qualificação do cuidado ao idoso. A atuação do farmacêutico no cuidado ao idoso, integrada com ações multidisciplinares, otimiza a farmacoterapia, com reflexos na segurança e efetividade da assistência prestada ao idoso.

\section{AGRADECIMENTOS}

À diretora clínica, Mônica Aparecida Costa, e à coordenadora técnica, Josiane Moreira da Costa, do Hospital Risoleta Tolentino Neves.

\section{REFERÊNCIAS}

1. Instituto Brasileiro de Geografia e Estatística. Sinopse dos resultados do Censo 2010. Pirâmide etária: distribuição da população por sexo, segundo os grupos de idade [acesso em 23 nov 2011]. Disponível em: <http://www.censo2010.ibge.gov.br/sinopse/ webservice $/$ default.php? $\operatorname{cod} 1=0 \& \operatorname{cod} 2=\& \operatorname{cod} 3=\& \mathrm{fr}$ $\mathrm{m}=$ piramide $>$.

2. Jyrkkä J, Enlund H, Korhonen MJ, Sulkava R, Hartikainen S. Patterns of drug use and factors associated with polypharmacy and excessive polypharmacy in elderly persons: results of the Kuopio 75+ study: a cross-sectional analysis. Drugs Aging 2009;26(6):493-503.

3. Rollason V, Vogt N. Reduction of polypharmacy in the elderly: a systematic review of the role of the pharmacist. Drugs Aging 2003;20(11):817-32.

4. Mion L, Odegard PS, Resnick B, Segal-Galan F; Geriatrics Interdisciplinary Advisory Group, American Geriatrics Society. Interdisciplinary care for older adults with complex needs: American Geriatrics Society position statement. J Am Geriatr Soc 2006;54(5):849-52.

5. Furtado JP. Arranjos Institucionais e Gestão da Clínica: princípios da Interdisciplinaridade e Interprofissionalidade. Cad Bras Saúde Mental 2009;1(1):1-11.

6. Badger N, Mullis S, Butler K, Tucker D. Pharmacist's intervention for older hospitalized patients. Am J Health Syst Pharm 2007;64(17):1794-6.

7. Spinewine A, Swine C, Dhillon S, Lambert P, Nachega JB, Wilmotte L, et al. Effect of a collaborative approach on the quality of prescribing for geriatric inpatients: a randomized, controlled trial. J Am Geriatr Soc 2007;55(5):658-65.

8. Chisholm-Burns MA, Kim Lee J, Spivey CA, Slack M, Herrier RN, Hall-Lipsy E, et al. US pharmacist's effect as team members on patient care: systematic review and meta-analyses. Med Care 2010;48(10):923-33.

9. Romano-Lieber NS, Teixeira JJV, Farhat FCLG, Ribeiro E, Crozatti MTL, Oliveira GSA. Revisão dos estudos de intervenção do farmacêutico no uso de medicamentos por pacientes idosos. Cad Saúde Pública 2002;18(6):1499-1507.

10. Brito GC, Menezes MS, Mesquita AR, Lyra Júnior DP. Efeito de um programa de manejo farmacoterapêutico em um grupo de idosos com hipertensão em Aracaju-Sergipe. Rev Ciênc Farm Básica Apl 2009;30(1):83-9.

11. Medeiros EL, Moraes CF, Karnikowski M, Nóbrega OT, Karnikowski MGO. Intervenção interdisciplinar enquanto estratégia para o uso racional de medicamentos em idosos. Ciênc Saúde Coletiva 2011;16(7):3139-49. 
12. Charlson M, Szatrowski TP, Peterson J, Gold J. Validation of a combined comorbidity index. J Clin Epidemiol 1994;47(11):1245-51.

13. Beers, MH. Explicit criteria for determining potentially inappropriate medication use by the elderly. An update. Arch Intern Med 1997; 157(14):1531-6.

14. Fick DM, Cooper JW, Wade WE, Waller JL, Maclean JR, Beers MH. Updating the Beers criteria for potentially inappropriate medication use in older adults: results of a US consensus panel of experts. Arch Intern Med 2003;163(22):2716-24.

15. Rudolph JL, Salow MJ, Angelini MC, McGlinchey $\mathrm{RE}$. The anticholinergic risk scale and anticholinergic adverse effects in older persons. Arch Intern Med 2008;168(5):508-13.

16. Chew ML, Mulsant BH, Pollock BG, Lehman ME, Greenspan A, Mahmoud RA, et al. Anticholinergic activity of 107 medications commonly used by older adults. J Am Geriatr Soc 2008;56(7):1333-41.

17. O'Mahony D, O'Connor MN. Pharmacotherapy at the end-of-life. Age Ageing 2011;40(4):419-22

18. Loganathan M, Singh S, Franklin BD, Bottle A, Majeed A. Interventions to optimise prescribing in care homes: systematic review. Age Ageing 2011;40(2):150-62.

19. Hanlon JT, Lindblad CI, Gray SL. Can clinical pharmacy services have a positive impact on drugrelated problems and health outcomes in communitybased older adults? Am J Geriatr Pharmacother 2004;2(1):3-13.

20. Davis RG, Hepfinger CA, Sauer KA, Wilhardt MS. Retrospective evaluation of medication appropriateness and clinical pharmacist drug therapy recommendations for home-based primary care veterans. Am J Geriatr Pharmacother 2007;5(1):40-7.

21. Lam S, Ruby CM. Impact of an interdisciplinary team on drug therapy outcomes in a geriatric clinic. Am J Health Syst Pharm 2005;62(6):626-9.

22. Dolder C, Szymanski B, Wooton T, McKinsey $\mathrm{J}$. Pharmacist interventions in an inpatient geriatric psychiatric unit. Am J Health Syst Pharm 2008;65(19):1795-6.

23. Gillespie U, Alassaad A, Henrohn D, Garmo $\mathrm{H}$, Hammarlund-Udenaes M, Toss $\mathrm{H}$, et al. A comprehensive pharmacist intervention to reduce morbidity in patients 80 years or older: a randomized controlled trial. Arch Intern Med 2009;169(9):894-900.

24. Storm A. Clinical Pharmacist at a geriatric ward. In: ESCP International Workshop on Geriatrics, Utrecht,The Netherlands, May 5-6, 2011. Int J Clin Pharm 2011;33(PSH-3):704.

25. Blix HS, Viktil KK, Moger TA, Reikvam A. Characteristics of drug-related problems discussed by hospital pharmacists in multidisciplinary teams. Pharm World Sci 2006;28(3):152-8.

26. Spinewine A, Dhillon S, Mallet L, Tulkens PM, Wilmotte L, Swine C. Implementation of ward-based clinical pharmacy services in Belgium - description of the impact on a geriatric unit. Ann Pharmacother 2006;40(4):720-8.

27. Loyola Filho AI, Uchoa E, Lima-Costa MF. Estudo epidemiológico de base populacional sobre uso de medicamentos entre idosos na Região Metropolitana de Belo Horizonte, Minas Gerais, Brasil. Cad Saúde Pública 2006;22(12):2657-67.

28. Kaur S, Mitchell G, Vitetta L, Roberts MS. Interventions that can reduce inappropriate prescribing in the elderly: a systematic review. Drugs Aging 2009;26(12):1013-28.

29. Uusvaara J, Pitkala KH, Kautiainen H, Tilvis RS, Strandberg TE. Association of anticholinergic drugs with hospitalization and mortality among older cardiovascular patients: a prospective study. Drugs Aging 2011;28(2):131-8.

30. Wawruch M, Macugova A, Kostkova L, Luha J, Dukat A, Murin J, et al. The use of medications with anticholinergic properties and risk factors for their use in hospitalised elderly patients. Pharmacoepidemiol Drug Saf 2012;21(2):170-6.

31. Cruciol-Souza JM, Thomson JC, Catisti DG. Avaliação de prescrições medicamentosas de um hospital universitário brasileiro. Rev Bras Educ Med 2008;32(2):188-96.

32. Braga TB, Pfaffenbach G, Weiss DP, Barros MB, Bergsten-Mendes G. Point prevalence of drug prescriptions for elderly and non-elderly inpatients in a teaching hospital. Sao Paulo Med J 2004;122(2):48-52.

33. Steinman MA, Handler SM, Gurwitz JH, Schiff GD, Covinsky KE. Beyond the prescription: medication monitoring and adverse drug events in older adults. J Am Geriatr Soc 2011;59(8):1513-20. 